\title{
Prognostic performance of clinical indices and model scorings for acute-on-chronic liver failure: A study of 164 patients
}

\author{
QIANQIAN ZHANG ${ }^{1}$, XIAOLIN GUO ${ }^{1}$, SHIXING ZHAO ${ }^{2}$, XIAOLI PANG ${ }^{1}$, \\ YANG WANG $^{1}$, YUJIAO ZHANG ${ }^{3}$ and BAORONG CHI ${ }^{1}$ \\ ${ }^{1}$ Department of Hepatobiliary and Pancreatic Diseases, The First Hospital of Jilin University, Changchun, Jilin 130021; \\ ${ }^{2}$ Department of Intensive Care Unit, Affiliated Hospital of Jining Medical University, Jining, Shandong 272000; \\ ${ }^{3}$ Department of Infectious Diseases, China-Japan Friendship Hospital, Beijing 100029, P.R. China
}

Received October 12, 2015; Accepted December 21, 2015

DOI: $10.3892 / \mathrm{etm} .2016 .3037$

\begin{abstract}
The present study aimed to analyze the prognostic factors of acute-on-chronic liver failure (ACLF), with the perspective of an improved selection of optimal therapeutic schemes. A retrospective analysis was used to study 164 patients with ACLF hospitalized between 2010 and 2014 in a single center. Patients were divided into favorable and unfavorable groups, according to the treatment outcomes. General characteristics and clinical manifestations were analyzed to determine whether they would affect the prognosis of the patients with ACLF, with a particular focus on the scoring systems Child-Pugh, model for end-stage liver disease (MELD), MELD with incorporation of sodium (MELD-Na), MELD and serum sodium ratio (MESO) and integrated MELD (iMELD). Hepatitis B virus infection was the predominant cause of ACLF, accounting for 88 cases (53.7\%). Age, prothrombin time, thrombin time, international normalized ratio (INR), prothrombin activity, serum sodium, albumin, total bilirubin, serum creatinine, platelets, fasting blood sugar, infections, hepatic encephalopathy, hepatorenal syndrome (HRS), and electrolyte disorder were revealed to be associated with prognosis. Age, serum sodium, INR, HRS, and infection were independent prognostic risk factors, as determined by multivariate analysis. Child-Pugh, MELD, MELD-Na, MESO and iMELD scoring systems all demonstrated adequate predictive values, with MELD-Na as the most effective scoring system. In conclusion, age, hyponatremia, INR, HRS and bacterial or fungal infections were reported to be independent prognostic risk factors for ACLF. Among the various liver function scoring systems, MELD-Na was the most accurate in predicting the prognosis of ACLF.
\end{abstract}

Correspondence to: Professor Baorong Chi, Department of Hepatobiliary and Pancreatic Diseases, The First Hospital of Jilin University, 71 Xinmin Street, Changchun, Jilin 130021, P.R. China E-mail: cbr0725@163.com

Key words: acute-on-chronic liver failure, prognosis, predictive value of tests, hepatic encephalopathy, hepatorenal syndrome

\section{Introduction}

Acute-on-chronic liver failure (ACLF) is characterized by an acute deterioration of liver function in patients with chronic liver disease, which is usually induced by a precipitating factor such as sepsis, alcohol or the rupture and bleeding of upper gastrointestinal varicose veins $(1,2)$. Occasionally, however, no specific precipitating event can be identified. Although the exact pathophysiology of ACLF development is unclear, unregulated inflammation is considered to be a major contributing factor (2). Furthermore, clinical manifestations are often characterized by severe gastrointestinal tract symptoms, rapidly increased jaundice, massive ascites, hepatic encephalopathy, hepatorenal syndrome (HRS), coagulopathy with severe bleeding tendency and rapidly progressing multi-organ failure, which may require liver transplantation (3).

Occurring in addition to pre-existing chronic liver diseases, ACLF is a deterioration syndrome characterized by the emergence of massive liver cell necrosis, accompanied by severe hepatic dysfunction with an associated mortality rate of up to $60-80 \%$ (4).

Due to severe functional liver impairment, patients with ACLF lack bioactive substances for life-sustaining activities and are affected by an accumulation of toxins (5), which causes multiple organ failure. Therefore, defining early and accurate prognostic factors for patients with ACLF is critically important when selecting an optimal treatment schedule. Scoring systems addressing the severity of liver disease, such as the Child-Pugh score (6) or the model for end-stage liver disease (MELD) (7), have been investigated. Indeed, Xun et al (8) reported that the integrated MELD (iMELD) and MELD with incorporation of sodium (MELD-Na) models predicted 3 -month mortality rates more accurately than traditional MELD. Furthermore, Shi et al (9) demonstrated that iMELD predicted hepatic-ACLF more accurately when compared with various other scoring systems (9). However, the prognostic assessment of ACLF in patients with multiple organ failure remains to be examined. The present study aimed to characterize patients with ACLF to facilitate the early recognition of the syndrome and to refine the prognostic assessment of ACLF. 


\section{Patients and methods}

Patients and primary endpoint. Patients with ACLF admitted to the First Hospital of Jilin University (Changchun, China) between June 2010 and June 2014 were retrospectively recruited. The definition of ACLF was based on the following Asia Pacific Association for Study of Liver criteria (10): Acute hepatic insult manifesting as jaundice and coagulopathy, complicated within 4 weeks by ascites and/or encephalopathy in a patient with previously diagnosed or undiagnosed chronic liver disease. Jaundice [ $\geq 5 \mathrm{mg} / \mathrm{dl}$ serum bilirubin $(85 \mu \mathrm{mol} / \mathrm{l})]$ and coagulopathy [international normalized ratio (INR) $>1.5$ or prothrombin activity (PTA) $<40 \%$ ] are mandatory for defining ACLF. Patients with hepatocellular carcinoma, arterial hypertension, coronary heart disease, diabetes and infectious diseases (with the exception of viral hepatitis) were excluded from the study.

In addition, the diagnosis of hepatic encephalopathy was based on the West Haven criteria (11). All medical treatments were recorded, including absolute bed rest, etiological (especially antiviral, lamivudine or entecavir) treatment, symptomatic treatment of complications, liver cell membrane protective agents, biliary stimulators, artificial liver support system, corticosteroid treatment and general supportive measures such as intravenous albumin and plasma.

Patients were divided into two groups according to their prognosis, namely favorable and unfavorable groups. These two subgroups were defined with reference to the primary endpoint of this study, which was hospital discharge or mortality. This investigation was approved by the Regional Ethics Committee of the First Hospital of Jilin University and all patients signed written informed consent forms.

Laboratory and clinical analysis. All patients with ACLF had fasting blood samples drawn within $24 \mathrm{~h}$ of admission for assessment of liver function. Blood samples were analyzed for levels of the following: Total bile acid; $\gamma$-globulins; prealbumin; thrombin time (TT); hemoglobin; serum sodium; alanine aminotransferase; aspartate aminotransferase; alkaline phosphatase; $\gamma$-glutamyltranspeptidase; albumin (ALB); total bilirubin (TBIL); cholinesterase; blood urea nitrogen; serum creatinine ( $\mathrm{SCr}$ ); prothrombin time (PT); international normalized ratio (INR); plasma prothrombin activity (PTA); white blood cell count; platelet count (PLT); fasting blood glucose (FBS); and $\alpha$-fetoprotein. Incidence of hepatic encephalopathy, bacterial or fungal infection, gastrointestinal bleeding, hepatorenal syndrome and electrolyte disturbance were also recorded. Hepatic encephalopathy is a clinical condition, characterized by the presence of cerebral dysfunction in patients with liver disease (11). To determine the presence of bacterial or fungal infections, laboratory examination of swab cultures was conducted. Infections included spontaneous peritonitis, pneumonia and intestinal, oral cavity and urinary tract infections. Gastrointestinal bleeding was determined by the presence of the symptoms of hematemesis or hematochezia. Hepatorenal syndrome was diagnosed as renal insufficiency (a plasma creatinine level $>1.5 \mathrm{mg} / \mathrm{dl}$ ) that progressed over days or weeks in the presence of severe liver disease, and in the absence of recognized nephrotoxic agents. Finally, electrolyte disturbance was assessed as two or more electrolyte
Table I. Etiological factors for acute-on-chronic liver failure.

\begin{tabular}{lc}
\hline Etiology & Cases $(\%)$ \\
\hline Hepatitis B infection & $88(53.7)$ \\
Hepatitis C infection & $3(1.8)$ \\
Alcoholic liver damage & $38(23.2)$ \\
Drug-induced liver damage & $5(3.0)$ \\
Auto-immune liver damage & $4(2.4)$ \\
Cryptogenic liver damage & $5(3.0)$ \\
Overlapping causes & $21(12.8)$
\end{tabular}

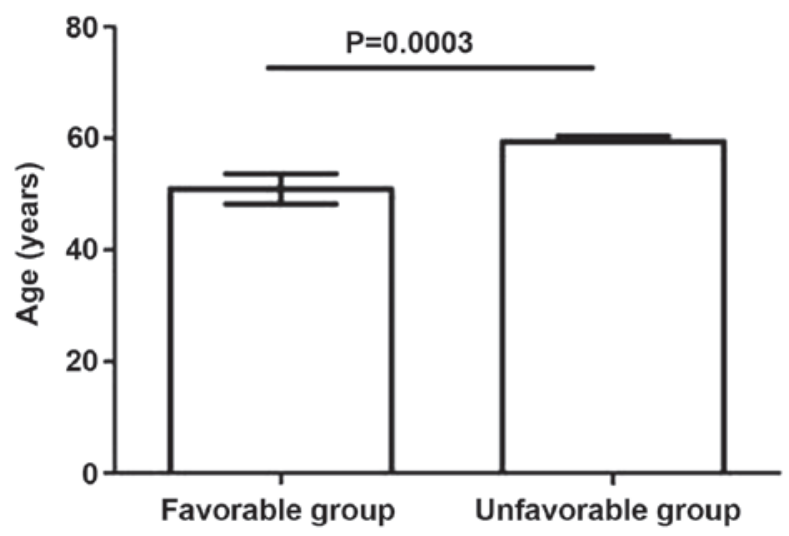

Figure 1. Association between age and prognosis. Data are presented as mean \pm standard deviation.

disorders, such as hyperkalemia and hyponatremia, occurring simultaneously.

Statistical analysis. All data are presented as mean values \pm standard deviation for continuous variables, and qualitative variables as proportions with percentages. The association between ACLF prognosis and biochemical indices or clinical complications was determined by univariate analyses, and the forward Wald approach was used for multivariate logistic regression models. Univariate analyses were as follows: A t-test, for normal distributions; a rank sum test, for non-normal distributions; and a chi-squared test, for comparison of complications between the groups. Furthermore, a comparative study of various liver function scoring systems, including Child-Pugh, MELD, MELD-Na, serum sodium ratio (MESO), and iMELD was performed $(6-8,12)$. To compare the predictive values of the various prognostic scoring systems, areas under the receiver operating curve (ROC) were calculated. Statistical analyses were performed using SPSS software version 18.0 (SPSS, Inc., Chicago, IL, USA). A P-value <0.05 was considered to indicate a statistically significant difference.

\section{Results}

Etiologies of ACLF. A total of 164 patients with ACLF were recruited into the study. Hepatitis $B$ virus (HBV) infection was the leading etiology of ACLF, followed by alcoholism, as shown in Table I. Overlapping causes accounted for the occurrence of ACLF in numerous patients, including 7 cases 
Table II. Comparison of serum biochemical parameters between the favorable and unfavorable subgroups.

\begin{tabular}{|c|c|c|c|}
\hline Parameters & $\begin{array}{c}\text { Favorable } \\
\text { group }(n=45)\end{array}$ & $\begin{array}{c}\text { Unfavorable } \\
\text { group }(n=119)\end{array}$ & P-value \\
\hline GLO, g/1 & $29.9 \pm 5.8$ & $33.7 \pm 7.9$ & 0.132 \\
\hline $\mathrm{TBA}, \mu \mathrm{mol} / 1$ & $200.8 \pm 115.3$ & $253.2 \pm 82.5$ & 0.063 \\
\hline $\mathrm{PA}, \mathrm{g} / \mathrm{l}$ & $0.12 \pm 0.04$ & $0.14 \pm 0.11$ & 0.564 \\
\hline $\mathrm{TT}, \mathrm{sec}$ & $21.8 \pm 2.6^{\mathrm{a}}$ & $26.6 \pm 4.4$ & $<0.001$ \\
\hline $\mathrm{HB}, \mathrm{g} / \mathrm{l}$ & $115.6 \pm 26.8$ & $111.2 \pm 29.5$ & 0.490 \\
\hline $\mathrm{Na}^{+}, \mathrm{mmol} / \mathrm{l}$ & $134.3 \pm 4.5^{\mathrm{a}}$ & $125.5 \pm 5.5$ & $<0.001$ \\
\hline ALT, IU/1 & $143.0(31.0-325.0)$ & $103.0(44.3-195.3)$ & 0.603 \\
\hline AST, IU/1 & $139.0(63.5-217.5)$ & $136.5(66.5-242.0)$ & 0.734 \\
\hline ALP, IU/1 & $117.0(93.0-168.5)$ & $122.5(94.5-169.7)$ & 0.938 \\
\hline GGT, IU/1 & $79.3(28.0-148.0)$ & $66.0(36.5-133.8)$ & 0.820 \\
\hline ALB, g/l & $28.1(27.0-32.1)^{\mathrm{a}}$ & $25.3(21.2-26.9)$ & $<0.001$ \\
\hline $\mathrm{TBIL}, \mu \mathrm{mol} / \mathrm{l}$ & $416.1\left(230.1-512.3^{\mathrm{a}}\right.$ & $501.3(352.8-656.1)$ & $<0.001$ \\
\hline CHE, IU/1 & $2463.3(1835.5-3933.5)$ & $2202.5(1635.3-2660.5)$ & 0.080 \\
\hline BUN, mmol/l & $4.8(3.7-5.6)$ & $6.5(3.6-11.8)$ & 0.071 \\
\hline $\mathrm{SCr}, \mu \mathrm{mol} / 1$ & $60.2(48.0-76.2)^{\mathrm{a}}$ & $98.0(78.3-151.6)$ & $<0.001$ \\
\hline PT, sec & $23.1(21.6-24.9)^{\mathrm{a}}$ & $28.8(23.1-35.6)$ & $<0.001$ \\
\hline INR & $1.7(1.6-2.0)^{\mathrm{a}}$ & $2.6(2.1-3.1)$ & $<0.001$ \\
\hline PTA, \% & $37.0(32.4-38.3)^{\mathrm{a}}$ & $31.0(22.9-37.0)$ & 0.026 \\
\hline WBC, $\times 10^{9} / 1$ & $7.4(4.7-9.9)$ & $6.7(4.8-11.9)$ & 0.605 \\
\hline PLT, $x 10^{9} / 1$ & $90.5(59.0-127.5)^{\mathrm{a}}$ & $68.5(52.3-79.8)$ & 0.025 \\
\hline FBS, mmol/1 & $4.9(4.2-5.6)^{\mathrm{a}}$ & $4.3(3.4-4.9)$ & 0.019 \\
\hline $\mathrm{AFP}, \mathrm{ng} / \mathrm{ml}$ & $15.1(3.5-152.2)$ & $16.7(3.87-59.3)$ & 0.922 \\
\hline
\end{tabular}

Comparison was conducted by a t-test method (means \pm standard deviation) for normal distribution conditions and a rank sum test (median and range) for non-normal distributions. ${ }^{\mathrm{a}} \mathrm{P}<0.05$ vs. the unfavorable group. GLO, $\gamma$-globulins; TBA, total bile acid; PA, prealbumin; TT, thrombin time; $\mathrm{HB}$, hemoglobin; $\mathrm{Na}^{+}$, serum sodium; ALT, alanine aminotransferase; AST, aspartate aminotransferase; ALP, alkaline phosphatase; GGT, $\gamma$-glutamyltranspeptidase; ALB, albumin; TBIL, total bilirubin; CHE, cholinesterase; BUN, blood urea nitrogen; SCr, serum creatinine; PT, prothrombin time; INR, international normalized ratio; PTA, plasma prothrombin activity; WBC, white blood cell count; PLT, platelet count; FBS, fasting blood glucose; and AFP, $\alpha$-fetoprotein.

of HBV infection associated with alcoholism, 6 cases of HBV associated with drug-induced liver damage, 4 cases of alcoholic liver damage associated with drug-induced liver damage, 2 cases of hepatitis $\mathrm{C}(\mathrm{HCV})$ infection associated with alcohol liver damage, 1 case of alcoholic liver damage associated with hepatitis E infection and 1 case of HCV infection associated with drug-induced liver damage.

Favorable and unfavorable group inclusion. A total of 45 individuals were included in the favorable group and 119 in the unfavorable group.

Associations of gender and age with prognosis. No statistically significant differences in prognosis were revealed to be associated with gender. Conversely, the results demonstrated that an older age was significantly $(\mathrm{P}<0.05)$ associated with a poorer prognosis. Logistic regression analysis demonstrated that age was an independent prognostic factor for ACLF $(\mathrm{P}<0.05$; Fig. 1).

Comparison of biochemical parameters between the two subgroups. Statistically significant differences between the favorable and unfavorable subgroups were observed in a number of biochemical parameters. Higher TT, TBIL, SCr, PT and INR values and lower serum sodium, ALB, PTA, PLT and FBS values were associated with a poor prognosis $(\mathrm{P}<0.05$; Table II). The other measured parameters revealed no significant change.

Comparison of complications between the two subgroups. The incidence of bacterial or fungal infection (including spontaneous peritonitis, pneumonia, intestinal infection, oral cavity fungal infection, and urinary tract infections), hepatic encephalopathy, HRS and electrolyte disturbance were significantly associated with the prognosis of patients with ACLF $(\mathrm{P}<0.05$; Table III).

Comparative data based on multivariate analysis. Logistic regression analysis identified that age, hyponatremia, INR HRS and bacterial or fungal infection were independent prognostic factors for ACLF (Table IV).

Comparison of liver function scoring systems between the two subgroups. The five scoring systems (Child-Pugh, MELD, 
Table III. Comparison of complications between the favorable and unfavorable subgroups [n (\%)].

\begin{tabular}{lcrrr}
\hline Complications & $\begin{array}{c}\text { Total } \\
\text { cases }(\mathrm{n}=164)\end{array}$ & $\begin{array}{c}\text { Favorable } \\
\text { group (n=45) }\end{array}$ & $\begin{array}{c}\text { Unfavorable } \\
\text { group (n=119) }\end{array}$ & $\begin{array}{r}\chi^{2} \\
\text { P-value }\end{array}$ \\
\hline Infection & $108(65.9)$ & $16(35.6)$ & $92(77.3)$ & 10.221 \\
HE & $63(38.4)$ & $8(17.8)$ & $55(46.2)$ & 6.023 \\
HRS & $42(25.6)$ & $5(11.1)$ & $37(31.1)$ & 10.829 \\
UGIB & $26(15.9)$ & $6(13.3)$ & $20(16.8)$ & 0.001 \\
Electrolyte disturbance & $122(74.3)$ & $20(44.4)$ & $102(85.7)$ & 0.012 \\
\end{tabular}

Infection, bacterial or fungal infection; HE, hepatic encephalopathy; HRS, hepatorenal syndrome; UGIB, upper gastrointestinal bleeding. Statistical analysis was conducted using a $\chi^{2}$ test.

Table IV. Logistic regression analysis of 164 patients with ACLF.

$95 \%$ CI of OR

\begin{tabular}{lccrrrrr}
\cline { 5 - 7 } Factors & $\mathrm{B}$ & $\mathrm{SE}$ & $\chi^{2}$ value & P-value & OR & Lower limit & Upper limit \\
\hline INR & 4.794 & 1.804 & 7.074 & 0.008 & 120.591 & 3.525 & 4.125 \\
Age & 0.225 & 0.088 & 6.769 & 0.007 & 1.253 & 1.057 & 1.486 \\
Hyponatremia & -0.389 & 0.128 & 9.348 & 0.002 & 0.679 & 0.527 & 0.871 \\
HRS & 1.096 & 0.002 & 4.621 & 0.031 & 2.511 & 1.533 & 4.702 \\
Infection & 2.934 & 1.289 & 5.196 & 0.024 & 18.827 & 1.507 & 234.838
\end{tabular}

CI, confidence interval; B, regression coefficient; SE, standard error; OR, odds ratio; ACLF, acute-on-chronic liver failure; INR, international normalized ratio; HRS, hepatorenal syndrome.

Table V. Comparison of liver function scoring systems between favorable and unfavorable subgroups (mean \pm standard deviation).

\begin{tabular}{lccc}
\hline Prediction models & $\begin{array}{c}\text { Favorable group } \\
(\mathrm{n}=45)\end{array}$ & $\begin{array}{c}\text { Unfavorable group } \\
(\mathrm{n}=119)\end{array}$ & P-value \\
\hline Child-Pugh & $11.3 \pm 1.2$ & $12.9 \pm 1.4$ & $<0.001$ \\
MELD & $19.8 \pm 4.4$ & $29.5 \pm 6.9$ & $<0.001$ \\
MELD-Na & $20.2 \pm 7.1$ & $43.5 \pm 13.9$ & $<0.001$ \\
MESO & $14.6 \pm 3.3$ & $23.2 \pm 5.9$ & $<0.001$ \\
iMELD & $40.4 \pm 6.3$ & $55.7 \pm 12.8$ & $<0.001$ \\
\hline
\end{tabular}

MELD, model for end-stage liver disease; MELD-Na, MELD with incorporation of sodium; MESO, MELD and serum sodium ratio; iMELD, integrated MELD.

MELD-Na, MESO and iMELD) produced results that were significantly different between the favorable and unfavorable groups $(\mathrm{P}<0.001$; Table $\mathrm{V})$. ROC curves were created to evaluate the predictive value of the five scoring systems. All scores provided good predictive values, with areas under the curves (AUCs) for the Child-Pugh, MELD, MELD-Na, MESO and iMELD scoring systems of $0.760,0.890,0.940,0.907$ and 0.860 , respectively. However, the MELD-Na score had a significantly higher predictive value compared with the other scoring systems (Table VI; Fig. 2).

\section{Discussion}

ACLF is a severe condition associated with various etiological factors (13), including viral infection, chronic alcohol abuse, use of illicit drugs and autoimmune liver disease. In the investigated cohort, the predominant causes of ACLF were HBV infection, chronic alcohol abuse, and various combinations of other etiologies. The prevailing role of $\mathrm{HBV}$ in the occurrence of ACLF was expected due to the high prevalence of HBV in China (14). Alcoholism and alternative combined etiologies 
Table VI. Optimal threshold, sensitivity, specificity, areas under the curve, and 95\% confidence interval of the five scoring systems for prognostic evaluation of patients with ACLF.

\begin{tabular}{lccccc}
\hline $\begin{array}{l}\text { Prediction } \\
\text { models }\end{array}$ & $\begin{array}{c}\text { Optimal } \\
\text { threshold }\end{array}$ & $\begin{array}{c}\text { Sensitivity } \\
(\%)\end{array}$ & $\begin{array}{c}\text { Specificity } \\
(\%)\end{array}$ & $\begin{array}{c}\text { Area under } \\
\text { the curve }\end{array}$ & $95 \%$ CI \\
\hline Child-Pugh & 12.6 & 0.625 & 0.811 & 0.760 & $(0.650,0.871)$ \\
MELD & 26.1 & 0.750 & 0.952 & 0.890 & $(0.819,0.961)$ \\
MELD-Na & 27.3 & 0.875 & 0.952 & 0.940 & $(0.868,1.000)$ \\
MESO & 18.2 & 0.858 & 0.906 & 0.907 & $(0.839,0.973)$ \\
iMELD & 48.3 & 0.750 & 0.952 & 0.860 & $(0.770,0.949)$ \\
\hline
\end{tabular}

ACLF, acute-on-chronic liver failure; MELD, model for end-stage liver disease; MELD-Na, MELD with incorporation of sodium; MESO, MELD and serum sodium ratio; iMELD, integrated MELD; CI, confidence interval.

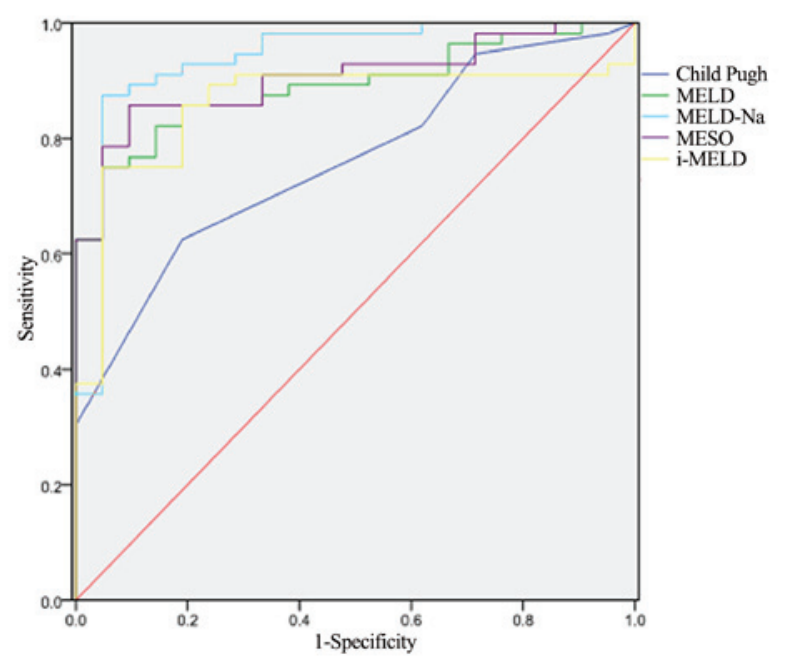

Figure 2. Evaluation of the predictive value of the scoring systems for prognostic evaluation of patients with ACLF. MELD, model for end-stage liver disease; MELD-Na, MELD with incorporation of sodium; MESO, MELD and serum sodium ratio; iMELD, integrated MELD.

are the two other main types of etiological factors contributing to the disease occurrence.

In the present study, patients with ACLF of older age groups, with higher PT, TT, INR, TBIL and SCr and lower serum sodium, PTA, ALB, PLT and FBS were more likely to have a poor prognosis. Complications such as bacterial or fungal infection, hepatic encephalopathy, HRS and electrolyte disturbance were also associated with poor prognosis. The results of the present investigation are concordant with those of Lal et al (15), who reported that high INR was an independent ACLF prognostic factor.

Cellular immunity is impaired in patients with ACLF, increasing the risk of infection and the infection-associated mortality rate (16). Accordingly, the results of this study illustrate a high frequency of infections (observed in 108 cases) and demonstrate that bacterial or fungal infections are an independent prognostic factor for ACLF.

Encephalopathy is another severe ACLF complication (17-19), and was observed in 63 of the cases analyzed $(38.4 \%)$. A number of the identified complications, including infection and electrolyte abnormalities, arise relatively suddenly, and may exacerbate the disturbances attributable to liver failure or exert a direct effect on the brain (20).

Upper gastrointestinal hemorrhage is a critical complication associated with ACLF; it is predominantly associated with high intravascular pressure, blood coagulation dysfunction and esophageal gastric varices, which exist prior to the occurrence of the bleeding (21). Through blood volume reduction, organ ischemia and hypoxia, multiple organ function failure may occur (22). Upper gastrointestinal bleeding was observed in $15.9 \%$ of the patients in the present study, and was not a prognostic factor.

HRS was identified as a strong prognostic risk factor within the present cohort. HRS corresponds to functional renal failure. In ACLF, renal vascular resistance increases progressively causing renal hypoperfusion. Furthermore, self-regulation of the renal perfusion function is also affected, and a small decrease in blood volume may lead to marked reduced renal perfusion, a further factor associated with kidney damage (23).

A total of 122 cases $(74.3 \%)$ of electrolyte disturbances, such as hyperkalemia and/or hyponatremia, were observed. These were predominantly associated with insufficient intake of food nutrients, vomiting, diarrhea, digestive disorders, long-term use of diuretics or a large amount of ascites drainage, long-term application of hypertonic glucose liquid and secondary aldosteronism. The present study demonstrated that the incidence of electrolyte disorder was significantly higher in the unfavorable group, as compared with the favorable group.

The most common type of electrolyte disturbances associated with patients with ACLF in the present study was hyponatremia, which was found to be an independent prognostic factor. Hyponatremia is primarily the result of solute-free water retention in liver cirrhosis. The proposed mechanism underlying this process is an association between the release of antidiuretic hormones and splanchnic arterial vasodilatation leading to reduced systemic vascular resistance (24-26).

The Child-Pugh score is the most commonly used evaluation system to assess hepatic reserve function in cirrhotic patients, facilitating evaluation of their prognosis. However, the Child-Pugh score, initially designed for patients with portosystemic shunt surgery, is associated with certain difficulties and inaccuracies $(27,28)$. The Child-Pugh classification 
uses TBIL, ALB, PT, presence of ascites and encephalopathy to reach a score. However, ALB, ascites and encephalopathy are subject to medical intervention. Furthermore, the Child-Pugh classification does not recognize a TBIL level $>51 \mu \mathrm{mol} / 1$. Additionally, ALB levels tend to be low immediately subsequent to bleeding or transfusions (29). The MELD scoring system was developed by the Mayo Clinic team, and was originally used to predict the prognosis of portal hypertension patients following transjugular intrahepatic portosystemic shunt (27). Kumar et al (30) reported that a MELD score that did not decrease by week 2 generated a $93.8 \%$ predictive chance of survival for the following 60 days. Ruf et al (31) demonstrated that, in ACLF, hyponatremia and the MELD score were risk factors that may affect the mortality of patients with liver failure, and supported the hypothesis that comprehensive serum sodium and a MELD score may provide a more accurate predictive approach. The MELD-Na model, established by Biggins et al (32), exhibited a more optimal predictive capacity than the MELD model. Using the MELD-Na model, Huo et al (12) established the MESO model, a non-invasive predictor of increased portal pressure in cirrhosis, which was superior to the MELD score in predicting patient mortality (12). Luca et al (33) proposed the addition of age and serum sodium concentrations to the MELD score system, resulting in the iMELD system, which allowed for enhancement of its predictive capacity. In the present study, all five scoring systems were validated as prognostic indicators for ACLF patients.

When establishing the accuracy of survival rate prediction at three months, the scoring systems with the better diagnostic or predictive value were those with the greater AUC of the ROC curve. In the present study, when considering that AUC $>0.7$ is the accepted threshold for clinical application; the five scoring systems demonstrated accurate predictive values for ACLF prognosis. Notably, the MELD-Na score was the most effective scoring system. Therefore, incorporating the serum sodium level into the MELD score would significantly improve the prediction accuracy of the prognosis of patients with ACLD.

In conclusion, age, hyponatremia, INR, HRS and bacterial or fungal infection were identified to be independent risk factors associated with ACLF prognosis. The MELD-Na score was the most efficient liver function evaluation system. The results of the present study may facilitate the prognostic assessment of patients with ACLF, and lead to improved overall management of this severe liver condition.

\section{References}

1. Fan HL, Yang PS, Chen HW, Chen TW, Chan DC, Chu CH, $\mathrm{Yu}$ JC, Kuo SM and Hsieh CB: Predictors of the outcomes of acute-on-chronic hepatitis B liver failure. World J Gastroenterol 18: 5078-5083, 2012.

2. Jalan R, Gines P, Olson JC, Mookerjee RP, Moreau R, Garcia-Tsao G, Arroyo V and Kamath PS: Acute-on chronic liver failure. J Hepatol 57: 1336-1348, 2012.

3. Wigg AJ, McCormick R, Wundke R and Woodman RJ: Efficacy of a chronic disease management model for patients with chronic liver failure. Clin Gastroenterol Hepatol 11: 850-858.e4, 2013.

4. Finkenstedt A, Nachbaur K, Zoller H, Joannidis M, Pratschke J, Graziadei IW and Vogel W: Acute-on-chronic liver failure: Excellent outcomes after liver transplantation but high mortality on the wait list. Liver Transpl 19: 879-886, 2013.
5. Katoonizadeh A, Laleman W, Verslype C, Wilmer A, Maleux G, Roskams $\mathrm{T}$ and Nevens F: Early features of acute-on-chronic alcoholic liver failure: A prospective cohort study. Gut 59: 1561-1569, 2010.

6. Lucey MR, Brown KA, Everson GT, Fung JJ, Gish R, Keeffe EB, Kneteman NM, Lake JR, Martin P, McDiarmid SV, et al: Minimal criteria for placement of adults on the liver transplant waiting list: A report of a national conference organized by the American Society of Transplant Physicians and the American Association for the Study of Liver Diseases. Liver Transpl Surg 3: 628-637, 1997.

7. Kamath PS, Wiesner RH, Malinchoc M, Kremers W, Therneau TM, Kosberg CL, D'Amico G, Dickson ER and Kim WR: A model to predict survival in patients with end-stage liver disease. Hepatology 33: 464-470, 2001.

8. Xun YH, Shi JP, Li CQ, Li D, Shi WZ, Pan QC, Guo JC and Zang GQ: Prognostic performance of a series of model for end-stage liver disease and respective $\Delta$ scores in patients with hepatitis B acute-on-chronic liver failure. Mol Med Rep 9: 1559-1568, 2014.

9. Shi Y, Yang Y, Hu Y, Wu W, Yang Q, Zheng M, Zhang S, $\mathrm{Xu} \mathrm{Z}, \mathrm{Wu} \mathrm{Y}$, Yan $\mathrm{H}$ and Chen Z: Acute-on-chronic liver failure precipitated by hepatic injury is distinct from that precipitated by extrahepatic insults. Hepatology 62: 232-242, 2015.

10. Sarin SK, Kumar A, Almeida JA, Chawla YK, Fan ST, Garg H, de Silva HJ, Hamid SS, Jalan R, Komolmit P, et al: Acute-on-chronic liver failure: Consensus recommendations of the Asian Pacific Association for the Study of the Liver (APASL). Hepatol Int 3: 269-282, 2009.

11. Ferenci P, Lockwood A, Mullen K, Tarter R, Weissenborn K and Blei AT: Hepatic encephalopathy - definition, nomenclature, diagnosis and quantification: Final report of the Working Party at the 11th World Congresses of Gastroenterology, Vienna, 1998. Hepatology 35: 716-721, 2002.

12. Huo TI, Wang YW, Yang YY, Lin HC, Lee PC, Hou MC, Lee FY and Lee SD: Model for end-stage liver disease score to serum sodium ratio index as a prognostic predictor and its correlation with portal pressure in patients with liver cirrhosis. Liver Int 27: 498-506, 2007.

13. Lee W, Squires RH Jr, Nyberg SL, Doo E and Hoofnagle JH: Acute liver failure: Summary of a workshop. Hepatology 47: 1401-1415, 2008

14. Ostapowicz G, Fontana RJ, Schiødt FV, Larson A, Davern TJ, Han SH, McCashland TM, Shakil AO, Hay JE, Hynan L, et al: Results of a prospective study of acute liver failure at 17 tertiary care centers in the United States. Ann Intern Med 137: 947-954, 2002.

15. Lal J, Thapa BR, Rawal P, Ratho RK and Singh K: Predictors of outcome in acute-on-chronic liver failure in children. Hepatol Int 5: 693-697, 2011.

16. Wasmuth HE, Kunz D, Yagmur E, Timmer-Stranghöner A, Vidacek D, Siewert E, Bach J, Geier A, Purucker EA, Gressner AM, et al: Patients with acute on chronic liver failure display 'sepsis-like' immune paralysis. J Hepatol 42: 195-201, 2005.

17. Crippin JS, Gross JB Jr and Lindor KD: Increased intracranial pressure and hepatic encephalopathy in chronic liver disease. Am J Gastroenterol 87: 879-882, 1992.

18. Jalan R, Dabos K, Redhead DN, Lee A and Hayes PC: Elevation of intracranial pressure following transjugular intrahepatic portosystemic stent-shunt for variceal haemorrhage. J Hepatol 27: 928-933, 1997.

19. Donovan JP, Schafer DF, Shaw BW Jr and Sorrell MF: Cerebral edema and increased intracranial pressure in chronic liver disease. Lancet 351: 719-721, 1998.

20. Olson JC, Wendon JA, Kramer DJ, Arroyo V, Jalan R, Garcia-Tsao G and Kamath PS: Intensive care of the patient with cirrhosis. Hepatology 54: 1864-1872, 2011.

21. Cremers I and Ribeiro S: Management of variceal and nonvariceal upper gastrointestinal bleeding in patients with cirrhosis. Therap Adv Gastroenterol 7: 206-216, 2014.

22. Bumaschny E, Doglio G, Pusajó J, Vetere L, Parra C, Grosso RM and Schieppati E: Postoperative acute gastrointestinal tract hemorrhage and multiple-organ failure. Arch Surg 123: 722-726, 1988.

23. Solís-Muñoz P: Acute on chronic liver failure and prognostic factors: Time for reevaluation. Rev Esp Enferm Dig 103: 169-176, 2011.

24. Wiesner R, Edwards E, Freeman R, Harper A, Kim R, Kamath P, Kremers W, Lake J, Howard T, Merion RM, et al: Model for end-stage liver disease (MELD) and allocation of donor livers. Gastroenterology 124: 91-96, 2003. 
25. Arroyo V and Colmenero J: Ascites and hepatorenal syndrome in cirrhosis: Pathophysiological basis of therapy and current management. J Hepatol 38 (Suppl 1): S69-S89, 2003.

26. Schrier RW, Gurevich AK and Cadnapaphornchai MA: Pathogenesis and management of sodium and water retention in cardiac failure and cirrhosis. Semin Nephrol 21: 157-172, 2001.

27. Malinchoc M, Kamath PS, Gordon FD, Peine CJ, Rank J and ter Borg PC: A model to predict poor survival in patients undergoing transjugular intrahepatic portosystemic shunts. Hepatology 31 : 864-871, 2000

28. Conn HO: A peek at the Child-Turcotte classification. Hepatology 1: 673-676, 1981 .

29. Al Sibae MR and Cappell MS: Accuracy of MELD scores in predicting mortality in decompensated cirrhosis from variceal bleeding, hepatorenal syndrome, alcoholic hepatitis, or acute liver failure as well as mortality after non-transplant surgery or TIPS. Dig Dis Sci 56: 977-987, 2011.
30. Kumar R, Krishnamoorthy TL, Tan HK, Lui HF and Chow WC Change in model for end-stage liver disease score at two weeks, as an indicator of mortality or liver transplantation at 60 days in acute-on-chronic liver failure. Gastroenterol Rep 3: 122-127, 2015.

31. Ruf AE, Kremers WK, Chavez LL, Descalzi VI, Podesta LG and Villamil FG: Addition of serum sodium into the MELD score predicts waiting list mortality better than MELD alone. Liver Transpl 11: 336-343, 2005.

32. Biggins SW, Kim WR, Terrault NA, Saab S, Balan V, Schiano T, Benson J, Therneau T, Kremers W, Wiesner R, et al: Evidence-based incorporation of serum sodium concentration into MELD. Gastroenterology 130: 1652-1660, 2006.

33. Luca A, Angermayr B, Bertolini G, Koenig F, Vizzini G, Ploner M, Peck-Radosavljevic M, Gridelli B and Bosch J: An integrated MELD model including serum sodium and age improves the prediction of early mortality in patients with cirrhosis. Liver Transpl 13: 1174-1180, 2007. 